\title{
Article \\ Atherogenic Index of Plasma and Its Association with Risk Factors of Coronary Artery Disease and Nutrient Intake in Korean Adult Men: The 2013-2014 KNHANES
}

\author{
Hye Ran Shin ${ }^{1}\left(\mathbb{D}\right.$, SuJin Song ${ }^{2} \mathbb{D}$, Jin Ah Cho ${ }^{1}$ and Sun Yung Ly ${ }^{1, *(\mathbb{D})}$ \\ 1 Department of Food and Nutrition, Chungnam National University, Daejeon 34134, Korea; \\ smile_hrshin@daum.net (H.R.S.); jacho@cnu.ac.kr (J.A.C.) \\ 2 Department of Food and Nutrition, Hannam University, Daejeon 34054, Korea; sjsong@hnu.kr \\ * Correspondence: sunly@cnu.ac.kr; Tel.: +82-42-821-6838; Fax: +82-42-821-8968
}

check for

updates

Citation: Shin, H.R.; Song, S.; Cho, J.A.; Ly, S.Y. Atherogenic Index of Plasma and Its Association with Risk Factors of Coronary Artery Disease and Nutrient Intake in Korean Adult Men: The 2013-2014 KNHANES. Nutrients 2022, 14, 1071. https:// doi.org/10.3390/nu14051071

Academic Editors: Siva Sankara Vara Prasad Sakamuri and Teresa Padro

Received: 28 December 2021

Accepted: 1 March 2022

Published: 3 March 2022

Publisher's Note: MDPI stays neutral with regard to jurisdictional claims in published maps and institutional affiliations.

Copyright: (C) 2022 by the authors. Licensee MDPI, Basel, Switzerland. This article is an open access article distributed under the terms and conditions of the Creative Commons Attribution (CC BY) license (https:// creativecommons.org/licenses/by/ $4.0 /)$.

\begin{abstract}
Coronary artery disease (CAD) has been linked to one of the highest death rates globally. The atherogenic index of plasma (AIP) may be an important predictor of atherosclerosis and cardiovascular disease, superior to the standard atherosclerotic lipid profile. This study investigated the relationship between AIP and obesity indices, blood glucose, lipid profile, and nutrient intake status in Korean adult men. The study included 1292 males aged $\geq 19$ years old who participated in the Korea National Health and Nutrition Examination Survey, 2013-2014. Participants were divided into four groups according to AIP quartiles, calculated as log (triglyceride (TG)/high-density lipoprotein cholesterol (HDL-C)). Body mass index, waist circumference, fasting blood glucose, hemoglobin A1c, total cholesterol, TG, and low-density lipoprotein cholesterol levels increased as AIP levels increased, whereas HDL-C level declined. As the level of AIP increased, intake of saturated fatty acid, calcium, phosphorus, riboflavin, milk, and dairy product decreased significantly, and the contribution rate of milk and dairy products to fat intake decreased. AIP was linked to obesity indices, blood glucose, and blood lipid profile in Korean men, suggesting that it could predict CAD.
\end{abstract}

Keywords: atherogenic index of plasma; coronary artery disease; vitamin D; calcium

\section{Introduction}

Coronary artery disease (CAD) has a high prevalence and incidence, and it is linked to one of the highest death rates in the world [1-3]. CAD is an atherosclerotic disease, which is inflammatory in nature, that manifests as stable angina, unstable angina, myocardial infarction, or sudden cardiac death [4]. Major risk factors for CAD include modifiable factors, such as high blood pressure, high blood cholesterol levels, smoking, diabetes, overweight or obesity, lack of physical activity, unhealthy diet, and stress, and the conventional factors, such as age (simply getting older increases risk), sex (men are generally at greater risk of coronary artery disease than females), family history, and race [5].

Serum lipid markers are major risk factors and predictors of CAD and cardiovascular disease (CVD) [2,6]. Dyslipidemia refers to abnormal levels of serum lipids (triglycerides (TG), high-density lipoprotein cholesterol (HDL-C), low-density lipoprotein cholesterol (LDL-C), and total cholesterol (TC)) [7,8]. Therefore, in clinical trials to date, high LDL-C, TG, TC, and low HDL-C have been reported to be highly related to CVD [9,10]. Based on this, there were various indices for predicting CVD, such as the TG/HDL-C ratio [11] and the atherogenic index (AI: LDL-C/HDL-C) [12]. Recently, the Atherogenic Index of Plasma (AIP) was introduced as a better marker for CAD than the conventional ratio in clinical studies [1,2,13-15].

The AIP, i.e., the logarithm of the value of TG divided by the value of plasma HDLC, correlates to LDL-C particle size and esterification rate in apoB-lipoprotein-depleted plasma and is a significant marker composed of TG and HDL-C [16]. AIP may be an 
important predictor of atherosclerosis and cardiovascular disease, superior to the standard atherosclerotic lipid profile [15]. Many studies have reported that elevated AIP values are positively correlated with diabetes $[17,18]$ and obesity indicators [15]. In addition, high AIP was associated with low blood vitamin D levels in men [19]. Although various studies on AIP are ongoing, domestic studies regarding AIP levels in Koreans are still scarce.

A large-scale study involving approximately 200,000 people in 27 countries for 10 years reported that men had a higher incidence of diagnosed CVD and higher mortality than women [4]. Hence, the male gender is a risk factor for CAD incidence, and mortality in men is thus high. As of 2020, the death rate due to heart diseases in South Korea is the second-highest, following malignant neoplasms among the causes of death, and is the number one cause of death by a single disease. The death rate due to heart diseases per 100,000 persons continuously increases yearly from $55.6 \%$ in 2015 to $63.0 \%$ in 2020 [20]. In addition, according to the Korea National Health Insurance Corporation, the proportion of the total population of Koreans receiving treatment for CAD was $58.9 \%$ men and $41.1 \%$ women in 2019. In particular, the medical expenditures for CAD in men increased by $56.2 \%$ compared to 2015 [21], indicating an urgent need for preventive measures against CAD in Korean men.

Meanwhile, it is conventionally known that a higher intake of saturated fatty acids is associated with CAD risk [22-24]. However, recent studies have reported that milk and dairy products containing saturated fats may prevent CAD $[25,26]$. Although those studies have not clearly identified how milk and dairy product intake reduces CVD, it is assumed that various factors, such as lactose and calcium, contained in milk and dairy products may prevent CAD. Therefore, it is thought that studies regarding the association between AIP, an indicator of $\mathrm{CAD}$, and dairy product intake in Koreans are required.

This study aimed to investigate the association between AIP and obesity, glucose metabolism, and lipid metabolism indicators in Korean adult men and analyze nutrient and food intakes according to AIP levels using data from the Korea National Health and Nutrition Examination Survey (KNHANES), 2013-2014, the last year in which blood vitamin D levels were measured in Korea.

\section{Materials and Methods}

\subsection{Participants}

The participants in this study were 12,092 persons aged $\geq 19$ years old, from 15,568 who participated in the first and second years of the sixth KNHANES (Figure 1). Among them, 8835 with incomplete information on nutrient and food intake $(n=1338)$ and related data $(n=7497)$ were excluded, and women $(n=1754)$, users of lipid lowering drugs $(n=80)$, and subjects with abnormal blood lipid levels ( $<1$ st percentile and $>99$ th percentile), $(n=131)$ were additionally excluded. Finally, data from 1292 adult men were used in the final analysis. The data from the KNHANES used in this study were obtained after receiving approval from the Institutional Review Board of the Korea Centers for Disease Control and Prevention in 2013-2014.

\subsection{General Characteristics}

Age, residential area, occupation, and education level were used as the general characteristic variables of the participants. Age was presented as a continuous variable, and residential area was classified into metropolitan areas (Seoul, Gyeonggi, and Incheon) and non-metropolitan areas. Occupation was classified according to the intensity of physical activity, which included office workers, outdoor workers, and unemployed. Educational level was divided into middle school graduation or below, high school graduation, and college graduation or higher. 


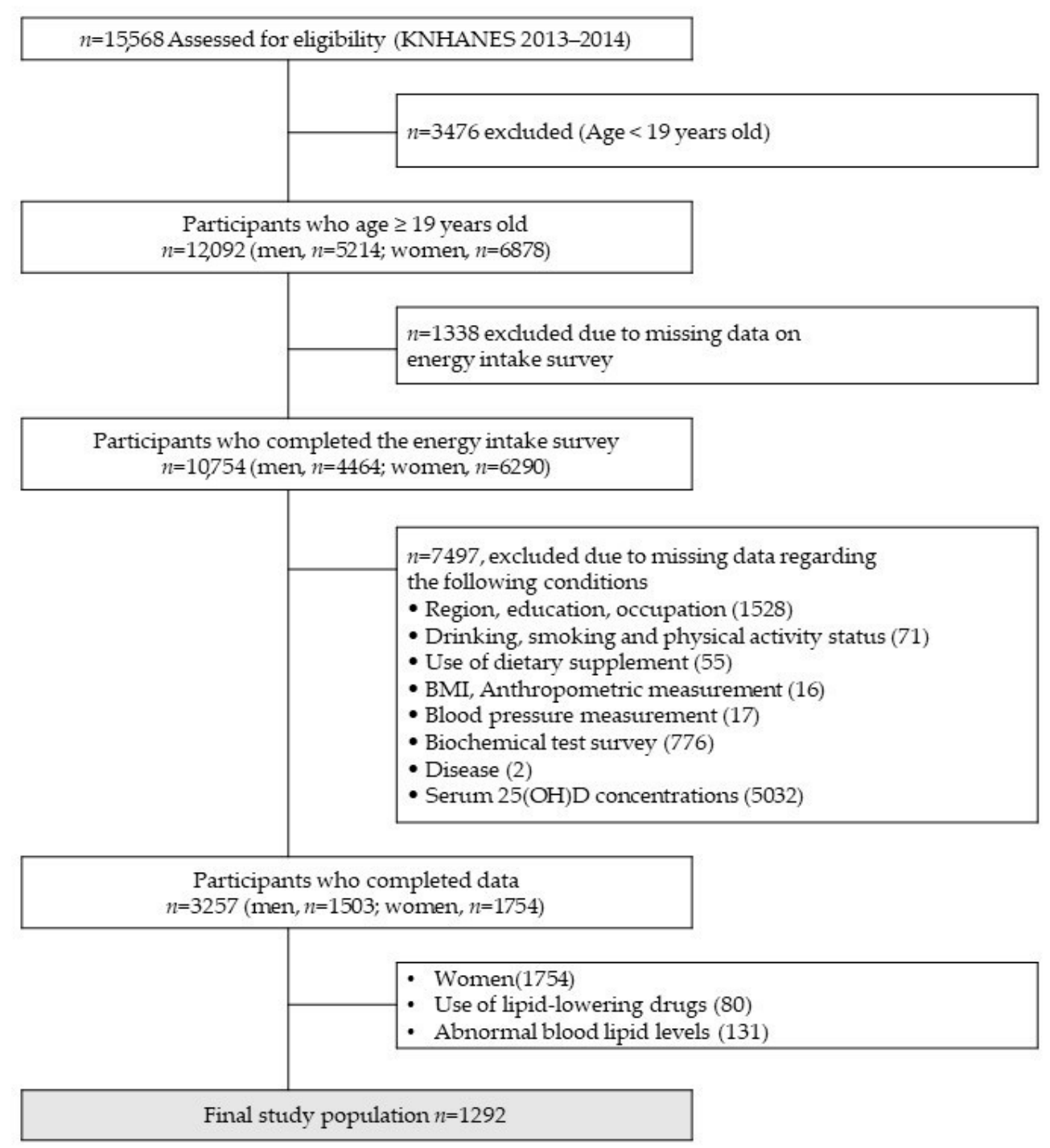

Figure 1. Flowchart of study participant inclusion and exclusion.

Drinking, smoking, physical activity, and dietary supplement intake were evaluated to examine the participants' lifestyle habits. In terms of drinking status, those who drank alcohol at least once a month for the past year were classified as 'yes'. For smoking status, those who were currently smoking were classified as 'yes'. For moderate-to-vigorous physical activity (MVPA), those who participated in at least one high-intensity physical activity (lifting or carrying something heavy, about $20 \mathrm{~kg}$ or greater, running, jumping rope, etc., for $20 \mathrm{~min}$ or longer per day for 1 week, three times per week), and/or moderate physical activity (performing physical activities, such as carrying light objects, walking briskly, or playing golf, for at least 30 min a day for 1 week, five times per week) were classified as 'yes'. In terms of dietary supplement intake, those who took a dietary supplement regardless of its type were classified as 'yes'.

For the disease prevalence rate, KNHANES variables of hypertension, dyslipidemia, stroke, myocardial infarction, angina, diabetes, and renal failure were used and classified into 'yes' and 'no'. In addition to the participants who currently answered 'yes', the following participants among the participants who answered 'no or do not know' were classified as 'yes': hypertension was defined as total systolic blood pressure of $\geq 140 \mathrm{mmHg}$ or diastolic blood pressure of $\geq 90 \mathrm{mmHg}$ [27]; dyslipidemia was defined as total cholesterol of $\geq 240 \mathrm{mg} / \mathrm{dL}$, LDL-cholesterol $\geq 160 \mathrm{mg} / \mathrm{dL}$, triglyceride $\geq 200 \mathrm{mg} / \mathrm{dL}$, HDL-cholesterol $<40 \mathrm{mg} / \mathrm{dL}$ [7]; and diabetes mellitus was defined as $\geq 6.5 \%$ of glycated hemoglobin or fasting blood sugar $\geq 126 \mathrm{mg} / \mathrm{dL}$ [28]. 


\subsection{Anthropometric Measurements and Biochemical Indicators}

The anthropometric measurements of the participants included height, weight, body mass index (BMI), and waist circumference. Height, weight, and waist circumference were measured in units of $0.1 \mathrm{~cm}, 0.1 \mathrm{~kg}$, and $0.1 \mathrm{~cm}$, respectively. BMI was calculated as weight $(\mathrm{kg})$ divided by height squared $\left(\mathrm{m}^{2}\right)$. Biochemical indicators, including fasting blood glucose and hemoglobin A1c (HbA1c) as indicators of glucose metabolism; TC, TG, HDL-C, and LDL-C as indicators of lipid metabolism; and blood 25-hydroxyvitamin $\mathrm{D}(25(\mathrm{OH}) \mathrm{D})$ were analyzed through blood tests after $\geq 8 \mathrm{~h}$ fasting. The TC, TG, HDL-C, and LDL-C values presented as $\mathrm{mg} / \mathrm{dL}$ values in the KNHANES were converted to $\mathrm{mmol} / \mathrm{L}$ as follows: $\mathrm{mg} / \mathrm{dL}$ was converted to the SI (in mmol/L) unit by multiplying the TC, LDL-C, HDL-C values by 0.02586 and TG in $\mathrm{mg} / \mathrm{dL}$ was converted to the SI unit, by multiplying the TG value by 0.01129 [29]. Men with $<1$ st percentile or $>99$ th percentile blood lipid levels of the same age group were considered abnormal and excluded by referring to the Reference Standard for Korean Health Index published by the National Health Insurance Sharing Service of Korea [30]. The atherogenic index of plasma (AIP) was calculated as a logarithmic transformation of the ratio of TG to HDL-C [16].

\subsection{Nutrient and Food Intake Survey}

In KNHANES, an experienced nutritionist interviewed the study participants and recorded what they ate for $24 \mathrm{~h}$ the previous day. Using the $24 \mathrm{~h}$ recall data from the KNHANES, this study evaluated the nutrient intake (energy, carbohydrates, fats, proteins, cholesterols, saturated fats, monounsaturated fatty acids, polyunsaturated fatty acids, omega-3 fatty acids, omega- 6 fatty acids, dietary fibers, calcium, phosphorus, iron, riboflavin, and the energy ratio of carbohydrate: protein: fat) and food intake by food group (grains, potatoes and starches, legumes and legume products, nuts and seed, vegetables, mushrooms, fruits, meat and poultries, eggs, fishes and shellfishes, seaweed, milk and dairy products, oils and fats, and beverages and alcohols).

Seven major lipid-rich food groups (meat and poultries, grains, oils and fat, milk and dairy products, legumes and legume products, eggs, and fishes and shellfish) and their contribution to fat intake were studied.

Milk and dairy products were classified into milk, ice cream, yogurt, cheese, and cream, and the intake amount of lipids ingested from the food is shown in Table S1. Condensed milk and milk were defined as milk, ice cream, ice milk, and sherbet as ice cream, and yogurt and liquid yogurt were defined and analyzed using the tertiary code of the $24 \mathrm{~h}$ recall method among the KNHANES survey data. Raw data were used for cheese and cream.

The nutrient intakes were calculated using the Standard Food Composition Table developed by the Rural Development Administration of the National Institute of Agricultural Sciences (eighth revision) [31]. The statistical analysis of nutrients intake and food group intake were presented as adjusted values for energy intake, age, BMI, smoking, and physical activity. The dietary reference intakes for Koreans in 2020 were used for dietary guidelines for Korean men [32].

\subsection{Statistical Analysis}

All statistical analyses were performed using SPSS version 26.0. The complex sample design of the KNHANES was reflected in the statistical analysis using weight, stratification (kstrata), and clustering (psu) variables. The continuous and categorical variables were presented as mean \pm standard error and $\mathrm{n}(\%)$, respectively. All participants were divided into quartile groups based on their AIP values $(<-0.38,-0.38-0.09,0.09-0.54, \geq 0.54)$. Testing for the significance of trend between the quartiles was presented as $p$-values for trend, using a complex sample general linear model (CSGLM). The general characteristics, except for age and lifestyle habits, were analyzed using cross-analysis of the complex samples. Using the complex samples descriptive statistics, age, anthropometric measures, and biochemical indicators were analyzed. Nutrient intake, with adjustment for energy intake, age, BMI, smoking, and physical activity, was calculated using complex samples, 
descriptive statistics, and CSGLM. The significance level in all statistical analyses was set to $p<0.05$.

\section{Results}

\subsection{General Characteristics of the Subjects According to Quartiles of AIP}

The mean age of the subjects in this study was 40.8 years, and the mean AIP was $<-0.38$ in $\mathrm{Q} 1,-0.38 \sim 0.09$ in $\mathrm{Q} 2,0.09 \sim 0.54$ in $\mathrm{Q} 3, \geq 0.54$ in $\mathrm{Q} 4$. The higher the quartile value of AIP, the higher the average age ( $p$ for trend $<0.001$, Table 1$)$. The number of smokers increased with increased AIP quartiles from Q1 to Q4 $(p<0.001)$, while the proportion of people with MVPA declined $(p<0.05)$. However, there was no difference in residential area, occupation, education level, and dietary supplement use according to the quartile of AIP.

Table 1. General characteristics of the subjects according to quartiles of AIP (Atherogenic Index of Plasma).

\begin{tabular}{|c|c|c|c|c|c|c|c|}
\hline & & \multicolumn{4}{|c|}{ Quartiles of AIP } & \multirow[b]{2}{*}{$\begin{array}{c}\text { Total } \\
(n=1292)\end{array}$} & \multirow[b]{2}{*}{$p$-Value } \\
\hline & & $\begin{array}{c}\mathrm{Q} 1 \\
(n=322) \\
<-0.38\end{array}$ & $\begin{array}{c}\mathrm{Q} 2 \\
(n=324) \\
-0.38 \text { to } 0.09\end{array}$ & $\begin{array}{c}\text { Q3 } \\
(n=323) \\
0.09 \text { to } 0.54\end{array}$ & $\begin{array}{c}\mathrm{Q} 4 \\
(n=323) \\
\geq 0.54\end{array}$ & & \\
\hline \multicolumn{2}{|c|}{ Age (years) } & $36.7 \pm 0.80$ & $40.7 \pm 0.78$ & $42.5 \pm 0.88$ & $43.1 \pm 0.81$ & $40.8 \pm 0.38$ & $<0.001^{(1)}$ \\
\hline \multirow{2}{*}{ Residential area } & Metropolitan & $154(51.5)$ & $156(51.2)$ & $171(55.2)$ & $176(57.9)$ & $657(53.8)$ & \multirow{2}{*}{0.374} \\
\hline & Non-metropolitan & $168(48.5)$ & $168(48.8)$ & $152(44.8)$ & $147(42.1)$ & $635(46.2)$ & \\
\hline \multirow{3}{*}{ Occupation } & Office workers & $86(27.1)$ & $90(29.8)$ & $99(36.1)$ & $107(36.0)$ & $382(32.1)$ & \multirow{3}{*}{0.242} \\
\hline & Outdoor workers & $161(49.7)$ & $159(46.2)$ & $149(42.4)$ & $152(19.6)$ & $289(22.1)$ & \\
\hline & Unemployed & $75(23.2)$ & $75(24.0)$ & $75(21.5)$ & $64(22.8)$ & $339(24.4)$ & \\
\hline \multirow{3}{*}{ Education level } & $<$ Middle school & $62(14.1)$ & $71(15.1)$ & $73(15.9)$ & $72(17.5)$ & $278(15.6)$ & \multirow{3}{*}{0.767} \\
\hline & High school & $132(46.2)$ & $129(43.7)$ & $120(39.9)$ & $124(41.5)$ & 505 (42.9) & \\
\hline & $\geq$ College & $128(39.7)$ & $124(41.1)$ & $130(44.2)$ & $127(40.9)$ & $509(41.4)$ & \\
\hline \multirow{2}{*}{ Drinking } & Yes & $249(80.0)$ & $231(70.9)$ & $243(75.8)$ & $248(76.8)$ & $971(75.9)$ & \multirow{2}{*}{0.096} \\
\hline & No & $73(20.0)$ & $93(29.1)$ & $80(24.2)$ & $75(23.2)$ & $321(24.1)$ & \\
\hline \multirow{2}{*}{ Smoking } & Yes & $101(31.0)$ & $116(36.6)$ & $150(48.4)$ & $163(53.7)$ & $530(40.9)$ & \multirow{2}{*}{$<0.001$} \\
\hline & No & $221(69.0)$ & $208(60.4)$ & $173(51.6)$ & $160(46.3)$ & $762(57.1)$ & \\
\hline \multirow{2}{*}{ MVPA $^{(2)}$} & Yes & $105(33.4)$ & $84(28.4)$ & $68(23.2)$ & $67(22.2)$ & $324(27.0)$ & \multirow{2}{*}{0.019} \\
\hline & No & $217(66.6)$ & $240(71.6)$ & $255(76.8)$ & $256(77.8)$ & $9686(73.0)$ & \\
\hline \multirow{2}{*}{ Dietary supplement } & Yes & $131(38.6)$ & $130(37.3)$ & $117(35.1)$ & $134(40.9)$ & $512(38.0)$ & \multirow{2}{*}{0.586} \\
\hline & No & $191(61.4)$ & $194(62.7)$ & $206(64.9)$ & 189 (59.1) & $780(62.0)$ & \\
\hline \multirow{2}{*}{ Hypertension } & Yes & $51(10.1)$ & $78(18.5)$ & $92(26.4)$ & $105(29.7)$ & $326(20.9)$ & \multirow[b]{2}{*}{$<0.001$} \\
\hline & No & $273(89.9)$ & $246(81.5)$ & $231(73.6)$ & $218(70.3)$ & $966(79.1)$ & \\
\hline \multirow{2}{*}{ Dyslipidemia } & Yes & $11(3.2)$ & $49(15.0)$ & $100(30.5)$ & $271(83.0)$ & $431(32.4)$ & \multirow{2}{*}{$<0.001$} \\
\hline & No & $311(96.8)$ & $275(85.0)$ & $223(69.5)$ & $52(17.0)$ & $861(67.6)$ & \\
\hline \multirow[b]{2}{*}{ Stroke } & Yes & $1(0.3)$ & $2(0.3)$ & $4(0.5)$ & $7(1.8)$ & $14(0.7)$ & \multirow[b]{2}{*}{0.034} \\
\hline & No & $321(99.7)$ & $322(99.7)$ & $319(99.5)$ & $316(98.2)$ & $1278(99.3)$ & \\
\hline \multirow{2}{*}{ Myocardial infarction } & Yes & - & $1(0.2)$ & $3(0.8)$ & $1(0.3)$ & $5(0.3)$ & \multirow{2}{*}{0.267} \\
\hline & No & $322(100)$ & $323(99.8)$ & $320(99.2)$ & $322(99.7)$ & 1287 (99.7) & \\
\hline \multirow{2}{*}{ Angina } & Yes & $4(0.8)$ & $1(0.1)$ & $3(0.4)$ & $4(1.1)$ & $12(0.6)$ & \multirow{2}{*}{0.178} \\
\hline & No & $318(99.2)$ & 323 (99.9) & $320(99.6)$ & 319 (98.9) & $1280(99.4)$ & \\
\hline \multirow{2}{*}{ Diabetes mellitus } & Yes & $15(2.6)$ & $27(6.5)$ & $30(7.4)$ & $53(13.5)$ & $125(7.4)$ & \\
\hline & No & 307 (97.4) & 297 (93.5) & 293 (92.6) & $270(86.5)$ & 1167 (92.6) & $<0.001$ \\
\hline & Yes & - & - & $1(0.3)$ & $2(0.4)$ & $3(0.2)$ & 0454 \\
\hline Renal failure & No & $322(100)$ & $324(100)$ & 322 (99.7) & 321 (99.6) & 1289 (99.8) & 0.454 \\
\hline
\end{tabular}

(1) $p$ for trend. (2) MVPA (moderate-to-vigorous physical activity): 'yes' for physical activity-moderate (for more than $30 \mathrm{~min}$ at a time and more than five times per week) and/or physical activity—high (for more than 20 min at a time and more than three times per week).

In the disease prevalence rate, hypertension, dyslipidemia, stroke, and diabetes increased from $\mathrm{Q} 1$ to $\mathrm{Q} 4(p<0.05)$. However, myocardial infarction, angina, and renal failure did not differ between the AIP quartiles.

\subsection{Anthropometric Measurements Data and Biochemical Indicator According to Quartiles of AIP}

As shown in Table 2, height decreased significantly as the AIP quartile increased ( $p$ for trend $<0.05$ ), while weight, BMI, and waist circumference increased significantly ( $p$ for trend $<0.001)$. As the AIP quartile increased, fasting blood glucose, HbA1c, TC, TG, and LDL-C levels increased significantly ( $p$ for trend $<0.001$ ), whereas HDL-C level decreased 
significantly ( $p$ for trend $<0.001$ ). Serum $25(\mathrm{OH}) \mathrm{D}$ was not significantly different according to the AIP.

Table 2. Anthropometric measurements data and biochemical indicators according to AIP quartiles.

\begin{tabular}{|c|c|c|c|c|c|c|}
\hline & \multicolumn{4}{|c|}{ Quartiles of AIP } & \multirow[b]{2}{*}{$\begin{array}{c}\text { Total } \\
(n=1292)\end{array}$} & \multirow[b]{2}{*}{$p$ for Trend } \\
\hline & $\begin{array}{c}\mathrm{Q} 1 \\
(n=322) \\
<-0.38\end{array}$ & $\begin{array}{c}\mathrm{Q} 2 \\
(n=324) \\
-0.38 \text { to } 0.09\end{array}$ & $\begin{array}{c}\mathrm{Q} 3 \\
(n=323) \\
0.09 \text { to } 0.54\end{array}$ & $\begin{array}{c}\mathrm{Q} 4 \\
(n=323) \\
\geq 0.54\end{array}$ & & \\
\hline Body mass index $\left(\mathrm{kg} / \mathrm{m}^{2}\right)$ & $22.5 \pm 0.18$ & $23.7 \pm 0.20$ & $25.0 \pm 0.20$ & $25.7 \pm 0.21$ & $24.27 \pm 0.10$ & $<0.001$ \\
\hline Waist circumference $(\mathrm{cm})$ & $78.0 \pm 0.50$ & $82.2 \pm 0.52$ & $85.1 \pm 0.52$ & $87.8 \pm 0.53$ & $83.48 \pm 0.26$ & $<0.001$ \\
\hline Fasting blood glucose (mg/dL) & $92.1 \pm 0.66$ & $95.6 \pm 1.12$ & $97.8 \pm 0.90$ & $104.3 \pm 1.39$ & $97.51 \pm 0.51$ & $<0.001$ \\
\hline HbA1c $(\%)$ & $5.54 \pm 0.03$ & $5.67 \pm 0.04$ & $5.72 \pm 0.03$ & $5.87 \pm 0.05$ & $5.70 \pm 0.02$ & $<0.001$ \\
\hline $\mathrm{TC}(\mathrm{mmol} / \mathrm{L})$ & $4.53 \pm 0.04$ & $4.79 \pm 0.05$ & $4.85 \pm 0.04$ & $5.08 \pm 0.05$ & $4.81 \pm 0.02$ & $<0.001$ \\
\hline $\mathrm{TG}(\mathrm{mmol} / \mathrm{L})$ & $0.70 \pm 0.01$ & $1.15 \pm 0.01$ & $1.62 \pm 0.02$ & $2.82 \pm 0.05$ & $1.57 \pm 0.01$ & $<0.001$ \\
\hline $\mathrm{HDL}-\mathrm{C}(\mathrm{mmol} / \mathrm{L})$ & $1.51 \pm 0.01$ & $1.31 \pm 0.01$ & $1.16 \pm 0.01$ & $1.07 \pm 0.01$ & $1.26 \pm 0.01$ & $<0.001$ \\
\hline $\mathrm{LDL}-\mathrm{C}(\mathrm{mmol} / \mathrm{L})$ & $2.87 \pm 0.04$ & $3.26 \pm 0.04$ & $3.36 \pm 0.04$ & $3.44 \pm 0.04$ & $3.23 \pm 0.02$ & $<0.001$ \\
\hline Serum $25(\mathrm{OH}) \mathrm{D}(\mathrm{ng} / \mathrm{mL})$ & $17.4 \pm 0.42$ & $17.3 \pm 0.48$ & $17.0 \pm 0.44$ & $16.8 \pm 0.40$ & $17.17 \pm 0.25$ & 0.216 \\
\hline AIP & $-0.79 \pm 0.02$ & $-0.13 \pm 0.01$ & $0.32 \pm 0.01$ & $0.93 \pm 0.02$ & $0.08 \pm 0.01$ & $<0.001$ \\
\hline
\end{tabular}

TC: Total Cholesterol; TG: Triglycerides; HDL-C: High-density lipoprotein cholesterol; LDL-C: Low-density lipoprotein cholesterol.

\subsection{Nutrient and Food Intake in Quartiles of AIP}

Nutrient intakes adjusted for energy intake, age, BMI, smoking, and physical activity by AIP quartiles are presented in Table 3. The intake of saturated fatty acid ( $p$ for trend $=0.21)$, calcium ( $p$ for trend $=0.001)$, phosphorus $(p$ for trend $=0.014)$, and riboflavin ( $p$ for trend $=0.002$ ) decreased significantly with the AIP quartile. There was no significant difference in the protein, polyunsaturated fatty acid, n-3 fatty acid, or dietary fiber intake. Dietary intakes from 14 food groups according to the AIP quartiles were analyzed after adjustment for confounders. Only milk and dairy products consumption decreased considerably from Q1 to Q4 $(p<0.05$, Table 4).

Table 3. Daily nutrient intake in quartiles of AIP (adjusted energy intake, age, BMI, smoking, and physical activity).

\begin{tabular}{|c|c|c|c|c|c|c|c|c|}
\hline & \multicolumn{5}{|c|}{ Quartiles of AIP } & \multirow[b]{2}{*}{$\begin{array}{c}\text { Total } \\
(n=1292)\end{array}$} & \multirow[b]{2}{*}{$p$-Value } & \multirow[b]{2}{*}{$\begin{array}{l}p \text { for } \\
\text { Trend }\end{array}$} \\
\hline & $\begin{array}{l}\text { Reference } \\
\text { Value for } \\
\text { KDIRs }{ }^{(1)}\end{array}$ & $\begin{array}{c}\mathrm{Q} 1 \\
(n=322) \\
<-0.38\end{array}$ & $\begin{array}{c}\mathrm{Q} 2 \\
(n=324) \\
-0.38 \text { to } 0.09\end{array}$ & $\begin{array}{c}\text { Q3 } \\
(n=323) \\
0.09 \text { to } 0.54\end{array}$ & $\begin{array}{c}\mathrm{Q} 4 \\
(n=323) \\
\geq 0.54\end{array}$ & & & \\
\hline Energy (kcal) & $1900-2600$ & $2561.8 \pm 66.7$ & $2623.0 \pm 79.3$ & $2561.2 \pm 72.8$ & $2620.6 \pm 70.6$ & $2591.7 \pm 41.2$ & 0.834 & 0.706 \\
\hline Carbohydrate (g) & 130 & $361.9 \pm 5.87$ & $362.2 \pm 6.18$ & $361.4 \pm 5.93$ & $346.1 \pm 6.75$ & $357.9 \pm 3.20$ & 0.221 & 0.089 \\
\hline Lipid (g) & - & $60.7 \pm 1.72$ & $58.4 \pm 1.99$ & $59.1 \pm 1.81$ & $57.1 \pm 1.59$ & $59.8 \pm 1.04$ & 0.469 & 0.456 \\
\hline Protein $(\mathrm{g})$ & $60-65$ & $88.2 \pm 1.55$ & $87.4 \pm 2.22$ & $87.7 \pm 1.72$ & $90.1 \pm 1.94$ & $88.4 \pm 1.02$ & 0.764 & 0.154 \\
\hline Cholesterol (mg) & $<300$ & $331.1 \pm 15.1$ & $327.9 \pm 16.7$ & $319.9 \pm 14.7$ & $321.7 \pm 18.2$ & $325.1 \pm 9.43$ & 0.948 & 0.643 \\
\hline Saturated fatty acid (g) & $\begin{array}{c}<7 \% \text { of } \\
\text { energy intake }\end{array}$ & $17.9 \pm 0.65$ & $17.2 \pm 0.73$ & $17.2 \pm 0.58$ & $15.8 \pm 0.57$ & $17.0 \pm 0.36$ & 0.076 & 0.021 \\
\hline Monounsaturated fatty acid (g) & - & $19.3 \pm 0.65$ & $19.0 \pm 0.82$ & $18.7 \pm 0.69$ & $17.7 \pm 0.61$ & $18.7 \pm 0.40$ & 0.300 & 0.075 \\
\hline Polyunsaturated fatty acid (g) & - & $14.8 \pm 0.56$ & $13.6 \pm 0.58$ & $14.6 \pm 0.61$ & $15.2 \pm 0.65$ & $14.6 \pm 0.33$ & 0.233 & 0.411 \\
\hline Omega-3 fatty acid $(\mathrm{g})$ & $1.4-1.6^{(2)}$ & $1.96 \pm 0.09$ & $1.73 \pm 0.89$ & $2.00 \pm 0.13$ & $2.14 \pm 0.14$ & $1.96 \pm 0.06$ & 0.058 & 0.127 \\
\hline Omega- 6 fatty acid (g) & $9-13^{(2)}$ & $13.0 \pm 0.51$ & $12.0 \pm 0.52$ & $12.7 \pm 0.53$ & $13.2 \pm 0.56$ & $12.7 \pm 0.29$ & 0.354 & 0.558 \\
\hline Dietary fiber $(\mathrm{g})$ & $25-30$ & $26.0 \pm 0.74$ & $25.9 \pm 0.70$ & $25.5 \pm 0.62$ & $25.1 \pm 0.62$ & $25.6 \pm 0.37$ & 0.800 & 0.329 \\
\hline Calcium (mg) & $700-800$ & $614.6 \pm 21.6$ & $569.3 \pm 18.7$ & $552.7 \pm 16.3$ & $520.8 \pm 17.6$ & $564.3 \pm 10.5$ & 0.012 & 0.001 \\
\hline Phosphorus (mg) & 700 & $1348.1 \pm 22.7$ & $1311.9 \pm 19.7$ & $1287.5 \pm 21.2$ & $1281.4 \pm 19.5$ & $1307.2 \pm 11.9$ & 0.089 & 0.014 \\
\hline Iron (mg) & $9-10$ & $19.1 \pm 0.67$ & $21.0 \pm 0.97$ & $21.1 \pm 1.28$ & $23.2 \pm 4.65$ & $21.1 \pm 1.50$ & 0.444 & 0.433 \\
\hline Riboflavin (mg) & $1.3-1.5$ & $1.80 \pm 0.49$ & $1.66 \pm 0.40$ & $1.64 \pm 0.41$ & $1.60 \pm 0.38$ & $1.67 \pm 0.23$ & 0.013 & 0.002 \\
\hline & \multicolumn{8}{|c|}{$\mathrm{C}: \mathrm{P}: \mathrm{F}$ ratio (\%) } \\
\hline $\mathrm{CHO}(\%)$ & $55-65$ & $62.8 \pm 0.67$ & $63.5 \pm 0.76$ & $0.63 \pm 0.69$ & $62.2 \pm 0.74$ & $62.9 \pm 0.38$ & 0.592 & 0.510 \\
\hline Protein (\%) & $7-20$ & $15.0 \pm 0.27$ & $15.0 \pm 0.36$ & $14.8 \pm 0.28$ & $15.7 \pm 0.31$ & $15.1 \pm 0.16$ & 0.145 & 0.111 \\
\hline Fat $(\%)$ & $5-30$ & $22.1 \pm 0.56$ & $21.4 \pm 0.58$ & $21.8 \pm 0.53$ & $21.9 \pm 0.59$ & $21.8 \pm 0.32$ & 0.804 & 0.953 \\
\hline
\end{tabular}

(1) Recommended intake. ${ }^{(2)}$ Adequate intake. 
Table 4. Food intake in quartiles of AIP (adjusted for energy intake, age, BMI, smoking, and physical activity).

\begin{tabular}{|c|c|c|c|c|c|c|}
\hline & \multicolumn{4}{|c|}{ Quartiles of AIP } & \multirow[b]{2}{*}{$\begin{array}{c}\text { Total } \\
(n=1292)\end{array}$} & \multirow[b]{2}{*}{$p$ for Trend } \\
\hline & $\begin{array}{c}\mathrm{Q} 1 \\
(n=322) \\
<-0.38\end{array}$ & $\begin{array}{c}\mathrm{Q} 2 \\
(n=324) \\
-0.38 \text { to } 0.09\end{array}$ & $\begin{array}{c}\mathrm{Q} 3 \\
(n=323) \\
0.09 \text { to } 0.54\end{array}$ & $\begin{array}{c}\mathrm{Q} 4 \\
(n=323) \\
\geq 0.54\end{array}$ & & \\
\hline Grains (g) & $329.2 \pm 9.20$ & $323.1 \pm 10.5$ & $333.7 \pm 9.55$ & $318.5 \pm 9.48$ & $326.1 \pm 5.31$ & 0.582 \\
\hline Potatoes and starches (g) & $49.8 \pm 9.04$ & $47.0 \pm 7.77$ & $39.5 \pm 6.43$ & $46.0 \pm 6.98$ & $45.6 \pm 4.74$ & 0.582 \\
\hline Legumes and legume products (g) & $41.6 \pm 5.43$ & $44.5 \pm 6.39$ & $48.4 \pm 6.02$ & $47.9 \pm 7.51$ & $45.6 \pm 3.08$ & 0.464 \\
\hline Nuts and seeds $(\mathrm{g})$ & $7.59 \pm 2.93$ & $9.14 \pm 2.38$ & $6.42 \pm 1.80$ & $5.61 \pm 1.05$ & $7.19 \pm 1.18$ & 0.349 \\
\hline Vegetables $(\mathrm{g})$ & $389.2 \pm 15.8$ & $383.3 \pm 13.2$ & $402.3 \pm 14.6$ & $376.8 \pm 14.1$ & $387.9 \pm 7.93$ & 0.758 \\
\hline Mushrooms (g) & $5.90 \pm 1.21$ & $6.27 \pm 1.17$ & $6.23 \pm 1.52$ & $7.46 \pm 1.53$ & $6.47 \pm 0.61$ & 0.496 \\
\hline Fruits $(\mathrm{g})$ & $157.8 \pm 14.0$ & $192.3 \pm 16.1$ & $168.1 \pm 16.5$ & $159.4 \pm 19.6$ & $169.4 \pm 9.03$ & 0.791 \\
\hline Meats and poultries (g) & $149.2 \pm 11.2$ & $174.2 \pm 14.2$ & $159.3 \pm 11.9$ & $150.0 \pm 10.4$ & $158.2 \pm 6.20$ & 0.788 \\
\hline Eggs (g) & $37.6 \pm 3.56$ & $34.1 \pm 3.10$ & $35.9 \pm 3.13$ & $36.8 \pm 4.25$ & $36.1 \pm 2.08$ & 0.980 \\
\hline Fish and shellfish (g) & $123.0 \pm 11.8$ & $104.7 \pm 9.82$ & $113.9 \pm 8.90$ & $113.9 \pm 8.90$ & $109.3 \pm 11.3$ & 0.524 \\
\hline Seaweed $(\mathrm{g})$ & $27.9 \pm 5.56$ & $20.6 \pm 5.40$ & $17.7 \pm 3.12$ & $25.2 \pm 6.31$ & $22.9 \pm 3.12$ & 0.684 \\
\hline Milk and dairy products (g) & $131.9 \pm 16.1$ & $84.90 \pm 9.74$ & $97.1 \pm 10.6$ & $85.7 \pm 12.3$ & $99.8 \pm 6.80$ & 0.044 \\
\hline Oils and fats $(\mathrm{g})$ & $11.9 \pm 0.83$ & $10.8 \pm 0.85$ & $11.4 \pm 0.92$ & $12.0 \pm 0.83$ & $11.5 \pm 0.45$ & 0.816 \\
\hline Beverages and alcohols (g) & $380.9 \pm 35.6$ & $535.7 \pm 55.5$ & $359.0 \pm 36.0$ & $460.4 \pm 38.9$ & $434.0 \pm 24.5$ & 0.680 \\
\hline
\end{tabular}

As a result of analyzing the contribution to fat intake of these seven major fat groups by AIP quartile, the amount of fat consumed from milk and dairy products decreased from the Q1 to the Q4 ( $p$ for trend < 0.01, Table 5).

Table 5. Contribution of seven major lipid-rich food groups toward the daily mean intake of lipid according to quartile of AIP (\%) (adjusted energy intake, age, BMI, smoking, and physical activity).

\begin{tabular}{|c|c|c|c|c|c|c|}
\hline & \multicolumn{4}{|c|}{ Quartiles of AIP } & \multirow[b]{2}{*}{$\begin{array}{c}\text { Total } \\
(n=1292)\end{array}$} & \multirow[b]{2}{*}{$p$ for Trend } \\
\hline & $\begin{array}{c}\mathrm{Q} 1 \\
(n=322) \\
<-0.38\end{array}$ & $\begin{array}{c}\mathrm{Q} 2 \\
(n=324) \\
-0.38 \text { to } 0.09\end{array}$ & $\begin{array}{c}\text { Q3 } \\
(n=323) \\
0.09 \text { to } 0.54\end{array}$ & $\begin{array}{c}\mathrm{Q} 4 \\
(n=323) \\
\geq 0.54\end{array}$ & & \\
\hline Meats and poultries & $25.5 \pm 2.04$ & $27.1 \pm 2.21$ & $23.9 \pm 2.01$ & $27.2 \pm 2.44$ & $25.9 \pm 1.14$ & 0.895 \\
\hline Grains & $18.1 \pm 1.70$ & $18.2 \pm 1.85$ & $19.4 \pm 1.80$ & $14.2 \pm 1.41$ & $17.5 \pm 0.93$ & 0.165 \\
\hline Oils and fats & $14.3 \pm 1.41$ & $13.7 \pm 1.33$ & $15.3 \pm 1.25$ & $17.3 \pm 1.48$ & $15.1 \pm 0.72$ & 0.116 \\
\hline Milk and dairy products & $9.10 \pm 1.48$ & $6.17 \pm 0.96$ & $6.26 \pm 1.04$ & $4.26 \pm 0.79$ & $6.45 \pm 0.65$ & 0.007 \\
\hline Legumes and legume products & $6.45 \pm 0.97$ & $6.60 \pm 1.05$ & $6.70 \pm 0.91$ & $5.03 \pm 1.06$ & $6.20 \pm 0.55$ & 0.338 \\
\hline Eggs & $5.85 \pm 0.73$ & $5.54 \pm 0.75$ & $5.15 \pm 0.64$ & $7.71 \pm 1.19$ & $6.06 \pm 0.49$ & 0.223 \\
\hline Fish and shellfish & $5.04 \pm 0.61$ & $4.56 \pm 0.61$ & $5.39 \pm 0.61$ & $4.88 \pm 0.71$ & $4.97 \pm 0.33$ & 0.889 \\
\hline
\end{tabular}

Table S1 show the lipid intake of subfoods in milk and dairy food according to quartile of AIP, which were adjusted for age energy intake, BMI, smoking and physical activity. Only milk and lipid intake from milk were significantly decreased from Q1 to Q4 ( $p$ for trend $<0.01)$.

\section{Discussion}

This study analyzed coronary artery disease risk factors according to the AIP quartile, using data on adult men extracted from the KNHANES. The AIP, calculated as $\log _{10}$ (TG/HDL-C), was initially constructed as a biomarker of plasma atherosclerosis. This value is designed to calculate small-dense LDL (sdLDL) [16]. The sdLDL has a high proportion of LDL and is small-sized, more sensitive to oxidative stress, and easily converted into oxidized LDL in the body, which causes inflammatory responses in the sub-endothelium of blood vessels and generates foam cells, leading to atherosclerosis [33]. However, sdLDL analysis has been difficult in clinical practice because it is expensive and requires a complex process. In comparison, AIP can be simply obtained, and accurately 
reflects sdLDL. Therefore, it has begun to be used as a predictive index for CAD [1]. It has been suggested that an AIP value $<0.11$ is associated with a low risk of CVD, and values between $0.11-0.21$ and $>0.21$ are associated with intermediate and increased risks, respectively [6].

This study found that low AIP in Korean adult men was favorable for body weight, waist circumference, BMI, fasting blood glucose, $\mathrm{HbA1c}$, and lipid metabolism parameters. Studies by Zhu et al. [15] and Kim [34], in which AIP levels were divided into quartiles as done in this study, reported that as AIP values increased from the first to fourth quartile, $\mathrm{BMI}$ values increased. In particular, the study by $\mathrm{Zhu}$ et al. found an association between AIP and obesity; when a BMI $\geq 28 \mathrm{~kg} / \mathrm{m}^{2}$ was defined as obesity, higher AIP levels were positively and strongly associated with obesity [15]. A study by Shen et al. regarding the correlation between AIP and waist circumference stated that an AIP between 0.12 and 0.21 or $>0.21$ indicated a possibility of borderline abdominal obesity or abdominal obesity, respectively, and that AIP can estimate abdominal obesity [35].

In addition, in this study, the prevalence of hypertension, dyslipidemia, stroke, and diabetes increased as the AIP quartile increased. It was suggested that AIP, which has already been verified as a predictor of CAD in many countries [13-15], could be a clinical indicator of vascular and metabolic diseases in Korea as well.

In this study, fasting blood glucose and $\mathrm{HbA1c}$ were positively associated with AIP. Furthermore, the prevalence of diabetes increased as the AIP quartile increased. A previous study involving Koreans reported a positive association between insulin resistance and fasting blood glucose levels, even in individuals with normal fasting blood glucose levels [36]. When insulin resistance occurs, it increases the breakdown of free fatty acids in adipose tissue, thereby increasing the amount of free fatty acids flowing into the liver and increasing the synthesis of fat and very-low-density lipoprotein in the liver while developing resistance to the action of insulin that activates lipoprotein lipase in the adipose tissue, which may result in increased blood triglycerides [37]. In addition, HbA1c increases cholesteryl ester transfer from HDL-C, causing a decrease in HDL-C [38]. Therefore, it can be said that there was a significant association between AIP and blood glucose index due to elevated TG and decreased HDL-C. A study by Suh et al. [39] found that the average LDL particle size was smaller and the proportion of sdLDL was higher in patients with type 2 diabetes mellitus (T2DM) compared to that in patients without T2DM [28]; therefore, it is thought that AIP, an indicator reflecting sdLDL, can predict T2DM.

In this study, from Q1 to Q4, the smoking rate decreased while the MVPA rate increased. Blood TG level could be elevated due to reduced physical activity [40] and excessive smoking [41,42]. This high TG value affected the high AIP. Many previous studies have shown that AIP has a positive association with TG, TC, and LDL-C and a negative association with HDL-C $[14,15,19]$. Traditionally, such lipid components have been used in the prediction of atherosclerotic CVDs, but AIP has the advantage of being a more relevant biochemical parameter than the conventional atherogenic index (AI) [12]. AIP was verified to be an indicator with clinical significance in long-term prognostic observation of diabetes and hypertension through a 9-year longitudinal study [13].

This study attempted to analyze the differences in nutrient and food intake in Korean adult men according to AIP quartiles. A significant difference was observed in saturated fatty acid, calcium, phosphorus, and riboflavin intake, and a higher AIP level was associated with lower intakes of these nutrients. Calcium is notable as an antihypertensive agent, contributing to the inverse association between calcium and CVD risk [43,44]. Inadequate riboflavin intake was associated with increased hypertension and low HDL-C [45].

However, this study found that saturated fat intake was relatively high in those with low AIP values, and it was, thus, necessary to analyze the food sources. The results of a comparison of nutrient intake by food group revealed that lower AIP values were associated with a greater intake of milk and dairy products. Saturated fat, calcium, phosphorus, and riboflavin are all nutrients found in large amounts in milk and dairy products, and their intake, in this study, was proportional to milk and dairy product intake. In addition, we 
found that as AIP increased, the contribution of fat intake from milk and dairy products decreased.

A study by Vimaleswaran et al. regarding milk intake and cardiometabolic disease outcomes reported that milk drinkers had a $14 \%$ lower risk of developing CAD than nonmilk drinkers [25]. Dehghan et al. reported that dairy product consumption was negatively associated with death risk due to CVDs or CVD risk. In that study, those who consumed two or more servings/day of total dairy products had a $22 \%$ lower risk of major CVD and a $23 \%$ lower cardiovascular mortality rate than those who did not consume any dairy products [26]. A multi-ethnic study regarding atherosclerosis reported that a higher intake of saturated fatty acids from dairy products was associated with a lower risk of overall mortality from atherosclerosis and cardiovascular events. That study also reported that a $5 \mathrm{~g} /$ day greater intake of milk saturated fatty acid was associated with a $21 \%$ lower risk of CVD, whereas a $5 \mathrm{~g}$ increase in meat saturated fat intake was associated with a $26 \%$ increased risk of CVD [3].

It can be expected that lactose in milk and dairy products may enhance calcium absorption and, thus, that calcium has beneficial effects on CAD. It was reported that when patients with CAD were given an additional intake of $50 \mathrm{~g}$ of lactose for 3 weeks, serum cholesterol levels were reduced [46]. Serum TG in mice fed both high fat and lactose was reported to be significantly lower than mice fed a simple high-fat diet [47]. Other potential mechanisms could be influenced by angiotensin-converting enzymes [48], osteocalcin [49] in milk and dairy products, and pathways of intestinal microbial activity, such as gut microbial fermentation [50]. The results of these previous studies suggest that milk and dairy product consumption may help prevent CAD. Our study supports the findings of these previous studies, but further studies are required to determine the mechanisms underlying the lower AIP values with increased consumption of milk and dairy products.

In this study, people with low AIP levels consumed more saturated fat through milk and dairy products; however, excessive saturated fat intake is still considered a major cause of heart disease. Excessive intake of saturated fatty acids is suggested as a potential risk factor for CAD and CVD by increasing LDL-C [22-24]. Therefore, the effect of saturated fatty acid intake on CAD according to food ingredients or food groups should be discussed in many studies.

Several epidemiologic and clinical studies show that the intake of omega-3 fatty acids, especially EPA and DHA, helps prevent cardiovascular disease [51-53]. In large-scale clinical studies targeting patients with cardiovascular disease or high-risk groups, EPA and DHA could reduce cardiovascular disease mortality [54]. In this study, the intake of omega-3 fatty acids was $1.85 \sim 2.05$, which was $\geq 1.4-1.6 \mathrm{~g}$, which is a sufficient intake for Koreans. In addition, the recommended intake ratio of omega-6:omega- 3 was 4-10:1, but in this study, it was about 8:1. Currently, it has been observed that omega-3 in Korean men is adequately maintained.

This study had several limitations. Since the results of this study were obtained through a cross-sectional study, it is difficult to derive a causal relationship between diet and AIP. In addition, because dietary data used in this study were based on a $24 \mathrm{~h}$ recall, they may not represent individuals' usual dietary intake. Although people taking lipidlowering drugs were excluded from the study subjects, it could not be ruled out that other metabolic diseases may affect the relationship between the intake of nutrients and food and CAD risk. However, the strengths of this study are that, to the best of our knowledge, this is the first study to assess the association between AIP and nutrients in Koreans.

This study found differences in coronary artery disease risk factor and nutrient intake according to AIP in Korean men. Those with a low AIP had favorable obesity, diabetes, and lipid metabolic indexes. Those with a low AIP had higher intakes of milk and dairy products, calcium, phosphorus, and riboflavin. AIP was negatively associated with saturated fat consumption attributed to milk and dairy product consumption, although there was no difference in total fat consumption among the AIP quartiles. Prospective studies are needed in the future to investigate the causal relationship between coronary artery disease 
risk factors and nutrient intake according to AIP. It is also necessary to expand the scope of studies to include both male and female genders for comparative analysis.

Supplementary Materials: The following supporting information can be downloaded at https:// www.mdpi.com/article/10.3390/nu14051071/s1: Table S1: Lipid intake of subfood in the group of milk and dairy products according to quartile of AIP (adjusted for energy intake, age, BMI, smoking, and physical activity).

Author Contributions: Conceptualization, H.R.S. and S.Y.L.; methodology, H.R.S. and S.S.; validation, H.R.S., J.A.C. and S.Y.L.; formal analysis, J.A.C. and S.Y.L.; investigation, J.A.C. and H.R.S.; resources, H.R.S.; data curation, S.S.; writing — original draft preparation, H.R.S. and S.S.; writing-review and editing, H.R.S., J.A.C. and S.Y.L.; visualization, H.R.S.; supervision, S.Y.L.; project administration, S.Y.L.; funding acquisition, S.Y.L. and H.R.S. All authors have read and agreed to the published version of the manuscript.

Funding: This research was funded by the National Research Foundation of Korea Grant funded by the Korean government (Ministry of Science and ICT), grant number 2020R1A2C1009608 and WISET-2021-293.

Institutional Review Board Statement: The study was conducted according to the guidelines of the Declaration of Helsinki and approved by the Research Ethics Review Committee of the Korea Centers for Disease Control and Prevention (approval number: 2013-07CON-03-4C, 2013-12EXP-03-5C).

Informed Consent Statement: Informed consent was obtained from all subjects involved in the study.

Data Availability Statement: 2013-2014 Sixth KNHANES data can be downloaded from the KNHANES homepage (https:/ / knhanes.kdca.go.kr/knhanes/eng/index.do, accessed on 1 March 2022).

Acknowledgments: The authors wish to thank the Korea Ministry of Health and Welfare and the Korea Disease Control and Prevention Agency for allowing their survey data to be analyzed in this study.

Conflicts of Interest: The authors declare no conflict of interest. The funders had no role in the study's design; in the collection, analyses, or interpretation of data; in the writing of the manuscript; or in the decision to publish the results.

\section{References}

1. Guo, Q.; Zhou, S.; Feng, X.; Yang, J.; Qiao, J.; Zhao, Y.; Shi, D.; Zhou, Y. The sensibility of the new blood lipid indicator-Atherogenic index of plasma (AIP) in menopausal women with coronary artery disease. Lipids Health Dis. 2020, 19, 27. [CrossRef] [PubMed]

2. Bora, K.; Pathak, M.S.; Borah, P.; Hussain, M.I;; Das, D. Association of the apolipoprotein A-I gene polymorphisms with cardiovascular disease risk factors and atherogenic indices in patients from Assam, Northeast India. Balk. J. Med. Genet. 2017, 20, 59-70. [CrossRef] [PubMed]

3. De Oliveira Otto, M.C.; Mozaffarian, D.; Kromhout, D.; Bertoni, A.G.; Sibley, C.T.; Jacobs, D.R., Jr.; Nettleton, J.A. Dietary intake of saturated fat by food source and incident cardiovascular disease: The multi-ethnic study of atherosclerosis. Am. J. Clin. Nutr. 2012, 96, 397-404. [CrossRef] [PubMed]

4. Walli-Attaei, M.; Joseph, P.; Rosengren, A.; Chow, C.K.; Rangarajan, S.; Lear, S.A.; AlHabib, K.F.; Davletov, K.; Dans, A.; Lanas, F; i et al. Variations between women and men in risk factors, treatments, cardiovascular disease incidence, and death in 27 high-income, middle-income, and low-income countries (PURE): A prospective cohort study. Lancet 2020, 396, 97-109. [CrossRef]

5. Hajar, R. Risk factors for coronary artery disease: Historical perspectives. Heart Views 2017, 18, 109. [CrossRef]

6. Dobiásová, M.; Frohlich, J.; Sedová, M.; Cheung, M.C.; Brown, B.G. Cholesterol esterification and atherogenic index of plasma correlate with lipoprotein size and findings on coronary angiography. J. Lipid Res. 2011, 52, 566-571. [CrossRef]

7. Rhee, E.-J.; Kim, H.C.; Kim, J.H.; Lee, E.Y.; Kim, B.J.; Kim, E.M.; Song, Y.; Lim, J.H.; Kim, H.J.; Choi, S. 2018 guidelines for the management of dyslipidemia in Korea. J. Lipid Atheroscler. 2019, 8, 78-131. [CrossRef]

8. AlQuaiz, A.M.; Kazi, A.; Youssef, R.M.; Alshehri, N.; Alduraywish, S.A. Association between standardized vitamin 25(OH)D and dyslipidemia: A community-based study in Riyadh, Saudi Arabia. Environ. Health Prev. Med. 2020, 25, 4. [CrossRef]

9. Sami Khaza, M. Atherogenic index of plasma (AIP) as a parameter in predicting cardiovascular risk in males compared to the conventional dyslipidemic indices (cholesterol ratios). Karbala J. Med. 2013, 6, 1506-1513.

10. Manninen, V.; Tenkanen, L.; Koskinen, P.; Huttunen, J.; Mänttäri, M.; Heinonen, O.; Frick, M.H. Joint effects of serum triglyceride and LDL cholesterol and HDL cholesterol concentrations on coronary heart disease risk in the Helsinki Heart Study. Implications for treatment. Circulation 1992, 85, 37-45. [CrossRef] 
11. Kim, M.R.; Jeong, S.J. Relationship between vitamin D level and lipid profile in non-obese children. Metabolites $2019,9,125$. [CrossRef] [PubMed]

12. Arnett, D.K.; Blumenthal, R.S.; Albert, M.A.; Buroker, A.B.; Goldberger, Z.D.; Hahn, E.J.; Himmelfarb, C.D.; Khera, A.; Lloyd-Jones, D.; McEvoy, J.W. 2019 ACC/AHA guideline on the primary prevention of cardiovascular disease: A report of the American college of cardiology/american heart association task force on clinical practice guidelines. J. Am. Coll. Cardiol. 2019, 74, e177-e232. [CrossRef] [PubMed]

13. Li, Y.-W.; Kao, T.-W.; Chang, P.-K.; Chen, W.-L.; Wu, L.-W. Atherogenic index of plasma as predictors for metabolic syndrome, hypertension and diabetes mellitus in Taiwan citizens: A 9-year longitudinal study. Sci. Rep. 2021, 11, 9900. [CrossRef]

14. Bo, M.S.; Cheah, W.L.; Lwin, S.; Moe Nwe, T.; Win, T.T.; Aung, M. Understanding the relationship between atherogenic index of plasma and cardiovascular disease risk factors among staff of an University in Malaysia. Nutr. Metab. 2018, $2018,7027624$. [CrossRef] [PubMed]

15. Zhu, X.; Yu, L.; Zhou, H.; Ma, Q.; Zhou, X.; Lei, T.; Hu, J.; Xu, W.; Yi, N.; Lei, S. Atherogenic index of plasma is a novel and better biomarker associated with obesity: A population-based cross-sectional study in China. Lipids Health Dis. 2018, 17, 37. [CrossRef]

16. Dobiášová, M.; Frohlich, J. The plasma parameter log (TG/HDL-C) as an atherogenic index: Correlation with lipoprotein particle size and esterification rate inapob-lipoprotein-depleted plasma (FERHDL). Clin. Biochem. 2001, 34, 583-588. [CrossRef]

17. Regmi, P.; Baral, B.; Raut, M.; Khanal, M. Atherogenic index of plasma for prediction of future cardiovascular disease in prediabetes and diabetes population. Atherosclerosis 2016, 252, e120. [CrossRef]

18. Scicali, R.; Giral, P.; D’Erasmo, L.; Cluzel, P.; Redheuil, A.; Di Pino, A.; Rabuazzo, A.M.; Piro, S.; Arca, M.; Beliard, S.; et al. High TG to HDL ratio plays a significant role on atherosclerosis extension in prediabetes and newly diagnosed type 2 diabetes subjects Diabetes Metab. Res. Rev. 2021, 37, e3367. [CrossRef]

19. Huang, F.; Liu, Q.; Zhang, Q.; Wan, Z.; Hu, L.; Xu, R.; Cheng, A.; Lv, Y.; Wang, L. Sex-specific association between serum vitamin $\mathrm{D}$ status and lipid profiles: A cross-sectional study of a middle-aged and elderly Chinese population. J. Nutr. Sci. Vitaminol. 2020, 66, 105-113. [CrossRef]

20. Statistics Korea. 2015 Annual Report on the Cause of Death Statistics. Available online: https://kostat.go.kr/portal/korea/kor $\mathrm{nw} / 1 / 6 / 2 /$ index.board? bmode=read\&bSeq=\&aSeq=385219\&pageNo=1\&rowNum=10\&navCount=10\&currPg=\&searchInfo= \&sTarget=title\&sTxt $=$ (accessed on 25 August 2021).

21. National Health Insurance Service. 940,000 Patients with Ischemic Heart Disease due to Cardiac Muscle Ischemia in 2019 Available online: https:/ / www.nhis.or.kr/nhis/together/wbhaea01600m01.do? mode=view\&articleNo=10805661\&article.offset= 0\&articleLimit=10\&srSearchVal=\%ED\%97\%88\%ED\%98\%88 (accessed on 8 November 2021).

22. Watts, G.F.; Jackson, P.; Mandalia, S.; Brunt, J.N.H.; Lewis, E.S.; Coltart, D.J.; Lewis, B. Nutrient intake and progression of coronary artery disease. Am. J. Cardiol. 1994, 73, 328-332. [CrossRef]

23. Hooper, L.; Martin, N.; Jimoh, O.F.; Kirk, C.; Foster, E.; Abdelhamid, A.S. Reduction in saturated fat intake for cardiovascular disease. Cochrane Database Syst. Rev. 2020. [CrossRef] [PubMed]

24. Hu, F.B.; Stampfer, M.J.; Manson, J.E.; Rimm, E.; Colditz, G.A.; Rosner, B.A.; Hennekens, C.H.; Willett, W.C. Dietary fat intake and the risk of coronary heart disease in women. N. Engl. J. Med. 1997, 337, 1491-1499. [CrossRef]

25. Vimaleswaran, K.S.; Zhou, A.; Cavadino, A.; Hyppönen, E. Evidence for a causal association between milk intake and cardiometabolic disease outcomes using a two-sample mendelian randomization analysis in up to 1,904,220 individuals. Int. J. Obes. 2021, 45, 1751-1762. [CrossRef]

26. Dehghan, M.; Mente, A.; Rangarajan, S.; Sheridan, P.; Mohan, V.; Iqbal, R.; Gupta, R.; Lear, S.; Wentzel-Viljoen, E.; Avezum, A.; et al. Association of dairy intake with cardiovascular disease and mortality in 21 countries from five continents (PURE): A prospective cohort study. Lancet 2018, 392, 2288-2297. [CrossRef]

27. Kim, H.C.; Ihm, S.-H.; Kim, G.-H.; Kim, J.H.; Kim, K.-i.; Lee, H.-Y.; Lee, J.H.; Park, J.-M.; Park, S.; Pyun, W.B. 2018 Korean society of hypertension guidelines for the management of hypertension: Part I-epidemiology of hypertension. Clin. Hypertens. 2019, 25, 1-6. [CrossRef]

28. Kim, M.K.; Ko, S.-H.; Kim, B.-Y.; Kang, E.S.; Noh, J.; Kim, S.-K.; Park, S.-O.; Hur, K.Y.; Chon, S.; Moon, M.K. 2019 clinical practice guidelines for type 2 diabetes mellitus in Korea. Diabetes Metab J. 2019, 43, 398-406. [CrossRef] [PubMed]

29. Haney, E.M.; Huffman, L.H.; Bougatsos, C.; Freeman, M.; Steiner, R.D.; Nelson, H.D. Screening and treatment for lipid disorders in children and adolescents: Systematic evidence review for the US Preventive Services Task Force. Pediatrics 2007, 120, e189-e214. [CrossRef] [PubMed]

30. National Health Insurance Service. The Reference Standard for Korean Health Index. Available online: https://nhiss.nhis.or.kr/ bd/ab/bdabf004lv.do (accessed on 24 February 2022).

31. Rural Development Administration National Institute of Agricultural Sciences. Standard Food Composition Table (8th Revision); Rural Development Administration National Institute of Agricultural Sciences: Wanju, Korea, 2013.

32. The Korean Nutrition Society. Dietary Reference Intakes for Koreans; The Korean Nutrition Society: Seoul, Korea, 2020.

33. Anber, V.; Griffin, B.A.; McConnell, M.; Packard, C.J.; Shepherd, J. Influence of plasma lipid and LDL-subfraction profile on the interaction between low density lipoprotein with human arterial wall proteoglycans. Atherosclerosis 1996, 124, 261-271. [CrossRef]

34. Kim, J.J.; Yoon, J.; Lee, Y.-J.; Park, B.; Jung, D.-H. Predictive value of the atherogenic index of plasma (AIP) for the risk of incident ischemic heart disease among non-diabetic Koreans. Nutrients 2021, 13, 3231. [CrossRef] [PubMed] 
35. Shen, S.-W.; Lu, Y.; Li, F.; Yang, C.-J.; Feng, Y.-B.; Li, H.-W.; Yao, W.-F.; Shen, Z.-H. Atherogenic index of plasma is an effective index for estimating abdominal obesity. Lipids Health Dis. 2018, 17, 11. [CrossRef]

36. Park, J.-M.; Kang, S.-M.; Lee, J.-W.; Lee, J.-W.; Kim, S.-W.; Lee, J.-H.; Cho, J.-B. Correlation between the ratio of triglyceride to high-density lipoprotein cholesterol and fasting glucose \& hemoglobin A1C. Korean J. Fam. Pract 2015, 5, 462-467.

37. Engin, A. The Definition and Prevalence of Obesity and Metabolic Syndrome. Adv. Exp. Med. Biol. 2017, 960, 1-17. [CrossRef] [PubMed]

38. Jung, S.-M.; Kim, E.-K.; Lee, H.-H.; Jung, K.-M.; Park, J.-W.; Ko, J.; Seo, H.-W.; Heo, T.-Y.; Jeong, B.-M. The relationship between smoking and hemoglobin A1c in adults without diabetes mellitus according to body mass index. Korean J. Fam. Pract. 2016, 6 , 339-345. [CrossRef]

39. Suh, S.; Park, H.-D.; Kim, S.W.; Bae, J.C.; Tan, A.H.-K.; Chung, H.S.; Hur, K.Y.; Kim, J.H.; Kim, K.-W.; Lee, M.-K. Smaller mean LDL particle size and higher proportion of small dense LDL in Korean type 2 diabetic patients. Diabetes Metab. J. 2011, 35, 536-542. [CrossRef] [PubMed]

40. Ofori, E.; Angmorterh, S. Relationship between physical activity, body mass index (BMI) and lipid profile of students in Ghana. Pan Afr. Med. J. 2019, 33. [CrossRef] [PubMed]

41. Koda, M.; Kitamura, I.; Okura, T.; Otsuka, R.; Ando, F.; Shimokata, H. The associations between smoking habits and serum triglyceride or hemoglobin A1c levels differ according to visceral fat accumulation. J. Epidemiol. 2016, 26, 208-215. [CrossRef]

42. Jain, R.B.; Ducatman, A. Associations between smoking and lipid/lipoprotein concentrations among US adults aged $\geq 20$ years. J. Circ. Biomark. 2018, 7, 1849454418779310. [CrossRef]

43. Van Mierlo, L.A.J.; Arends, L.R.; Streppel, M.T.; Zeegers, M.P.A.; Kok, F.J.; Grobbee, D.E.; Geleijnse, J.M. Blood pressure response to calcium supplementation: A meta-analysis of randomized controlled trials. J. Hum. Hypertens. 2006, 20, 571-580. [CrossRef]

44. Kong, S.H.; Kim, J.H.; Hong, A.R.; Cho, N.H.; Shin, C.S. Dietary calcium intake and risk of cardiovascular disease, stroke, and fracture in a population with low calcium intake. Am. J. Clin. Nutr. 2017, 106, 27-34. [CrossRef]

45. Ahn, J.Y.; Kim, I.S.; Lee, J.-S. Relationship of riboflavin and niacin with cardiovascular disease. Korean J. Clin. Lab. Sc 2019, 51, 484-494. [CrossRef]

46. Agarwal, B.; Sikka, K.; Srivastava, D.; Wali, M. Effect of lactose on serum lipids in cases of coronary artery disease. J. Indian Med. Assoc. 1980, 75, 153-156. [PubMed]

47. Goseki-Sone, M.; Maruyama, R.; Sogabe, N.; Hosoi, T. Effects of dietary lactose on long-term high-fat-diet-induced obesity in rats Obesity 2007, 15, 2605-2613. [CrossRef] [PubMed]

48. Pörsti, I.; Fan, M.; Kööbi, P.; Jolma, P.; Kalliovalkama, J.; Vehmas, T.I.; Helin, H.; Holthöfer, H.; Mervaala, E.; Nyman, T.; et al. High calcium diet down-regulates kidney angiotensin-converting enzyme in experimental renal failure. Kidney Int. 2004, 66, 2155-2166. [CrossRef] [PubMed]

49. Choi, H.J.; Yu, J.; Choi, H.; An, J.H.; Kim, S.W.; Park, K.S.; Jang, H.C.; Kim, S.Y.; Shin, C.S. Vitamin K2 supplementation improves insulin sensitivity via osteocalcin metabolism: A placebo-controlled trial. Diabetes Care 2011, 34, e147. [CrossRef] [PubMed]

50. St-Onge, M.-P.; Farnworth, E.R.; Jones, P.J.H. Consumption of fermented and nonfermented dairy products: Effects on cholesterol concentrations and metabolism. Am. J. Clin. Nutr. 2000, 71, 674-681. [CrossRef]

51. Geleijnse, J.M.; Giltay, E.J.; Grobbee, D.E.; Donders, A.R.; Kok, F.J. Blood pressure response to fish oil supplementation: Metaregression analysis of randomized trials. J. Hypertens. 2002, 20, 1493-1499. [CrossRef]

52. Mozaffarian, D.; Micha, R.; Wallace, S. Effects on coronary heart disease of increasing polyunsaturated fat in place of saturated fat: A systematic review and meta-analysis of randomized controlled trials. PLoS Med. 2010, 7, e1000252. [CrossRef]

53. Harris, W.S.; Kris-Etherton, P.M.; Harris, K.A. Intakes of long-chain omega-3 fatty acid associated with reduced risk for death from coronary heart disease in healthy adults. Curr. Atheroscler. Rep. 2008, 10, 503-509. [CrossRef]

54. Lavie, C.J.; Milani, R.V.; Mehra, M.R.; Ventura, H.O. Omega-3 polyunsaturated fatty acids and cardiovascular diseases. J. Am. Coll. Cardiol. 2009, 54, 585-594. [CrossRef] 